\title{
The one and the many: a case highlighting comorbidity and complexity in psychiatry
}

\author{
Jonathan Williams, ${ }^{1}$ Ruth Cain, ${ }^{2}$ Danilo Arnone, ${ }^{3,4}$ (1) Michalis Kyratsous ${ }^{4,5}$ (1)
}

BJPsych Bulletin (2020) 44, 169-173, doi:10.1192/bjb.2020.23

'Guy's and St Thomas' General Practitioner Vocational Training Scheme, London; ${ }^{2}$ University of Kent; ${ }^{3}$ Department of Psychiatry, College of Medicine and Health Sciences, United Arab Emirates University; ${ }^{4}$ Department of Psychological Medicine, Institute of Psychiatry, Psychology \& Neuroscience, King's College London; 5 South London and Maudsley NHS Foundation Trust

Correspondence to Michalis Kyratsous (michalis.1.kyratsous@kcl.ac.uk)

First received 12 Jan 2019, final revision 9 Feb 2020, accepted 18 Feb 2020

(c) The Authors 2020. This is an Open Access article, distributed under the terms of the Creative Commons Attribution licence (http://creativecommons.org/ licenses/by/4.0/), which permits unrestricted re-use, distribution, and reproduction in any medium, provided the original work is properly cited.
Diagnostic categorisation is a typical stage of the medical model. Nevertheless, it is important to consider what is helpful to both the clinician and the patient when symptoms, experiences and perceptions are categorised. In this case report, we address the problem of comorbidity and complexity in psychiatry. Research and clinical experience point to significant overlap between personality disorders, mood disorders, and developmental disorders such as attention-deficit hyperactivity disorder. In the face of such complexity, we discuss ways of addressing and managing multiple diagnoses in clinical practice. We synthesise the perspectives and views of a general practice trainee, two consultant psychiatrists and a person with lived experience.

Declaration of interest D.A. has received travel grants from Jansen-Cilag and Servier and sponsorship from Lundbeck.

Keywords Comorbidity; complexity; pluralism; co-production; phenomenology.

\section{Clinical scenario}

A community-dwelling 45-year-old lady presented to secondary care services (mood, anxiety and personality clinic). Established previous diagnoses included attention-deficit hyperactivity disorder (ADHD), bipolar affective disorder (BPAD) and emotionally unstable personality disorder (EUPD).

At the time of the first interview she presented with multiple difficulties, evidence of minimal response to pharmacological treatments and historical difficulty engaging with psychological services. The most distressing complaint involved a recent episode lasting around 2 months when she had felt elated and more impulsive, with an increased level of activity and risk-taking behaviours. During the episode she had travelled abroad and become involved in a short-term relationship with a much younger man who was financially exploitative and had a history of criminal behaviour. She also reported experimenting with different illegal substances and incurring significant financial loss after she had lent a large sum of money. The experience as a whole had a negative and distressing effect on her life. She reported feelings of guilt and shame, which resulted in her feeling low in mood, but with some variability. On examination, she was objectively euthymic with a reactive and appropriate affect. There was also a hint of cognitive acceleration, which she described as speeding and agitated thoughts with no useful purpose, observed as slight pressure of speech.
Her risk fluctuated throughout the months she spent with the team. At times, she demonstrated an ability to think and plan ahead with strong protective factors, but also reporting distressing thoughts of self-harm, which she had not acted on.

\section{Initial diagnostic considerations}

Diagnostic features suggestive of a primary mood disorder ${ }^{1}$ included elation, increased physical and mental activity, and overspending, all of which appeared to occur during a distinct episode, resulting in a severe or complete disruption of normal life with cognitive acceleration on examination. However, there were also features suggestive of EUPD - presenting in emotional crisis following an episode which could be interpreted as 'impulsive', i.e. without consideration of the consequences and possibly due to lack of self-control involving an intense relationship along with suicidal ideation. Overactivity and impaired inattention of early onset, evident in more than one situation, are cardinal features of hyperkinetic disorder (attention deficit) and could have also explained some of the behaviours and symptoms experienced during this episode. It would be a plausible hypothesis that with an established diagnosis of ADHD, changes in mood might have exacerbated symptoms of ADHD, resulting in a similar behaviour.

There were many overlapping features of each condition; in this case including impulsivity, overactivity, disinhibition, 
mood instability, lack of self-control, relationship difficulties, and thoughts of self-harm and suicide. One of the main difficulties when dealing with such complexity is trying to establish whether each symptom is a core feature of a disorder or the result of a combination of features of different disorders. The consideration of both the aetiological nature and severity are crucial elements of the design of the treatment.

\section{Questions regarding her presentation}

- Is the above psychopathology the result of one psychiatric disorder or a combination of conditions with overlapping features?

- What should be the focus of the follow-up sessions?

\section{Summary box}

- Diagnostic categorisation is a typical stage of the medical model. Nevertheless, it is important to consider what is helpful to both the patient and the clinician when symptoms and experiences are categorised.

- What can improve diagnostic specificity with conditions such as EUPD, ADHD and BPAD, which have significant phenotypic overlap? Is it really true that many different diagnostic entities can coexist, or are we rather describing one dynamic cluster of situation-specific, overlapping phenotypic variants?

- Patients' symptoms and experiences can be communicated and interpreted differently based on factors such as cognitive state, social and cultural background, current mental state, etc. Can we ever fully understand what the patient is experiencing?

- How do we use phenomenology to make hypotheses and formulate clinical problems?

- How do we practically approach complexity and comorbidity in community psychiatry?

\section{The usefulness and limits of diagnostic categories}

Criticism of standard psychiatric taxonomy remains vigorously debated and theoretically unresolved. ${ }^{2,3}$ Interrater agreement statistics for psychiatric diagnostic clarification remain poor, despite DSM and ICD revisions, and comorbidity rates within psychiatry are high, which could be reflective of this. ${ }^{2}$

A categorical approach to diagnosis serves a valid purpose. The presence or absence of symptoms helps to formulate diagnoses and informs clinical management. ${ }^{3,4}$ It offers objective, operationalised criteria allowing for observation, analysis, research and effective communication of information, but it also relies heavily on accurate information gathering, interpretation and diagnosis, all of which can be influenced by many factors. An assessment based purely on operationalised criteria neglects many of the subtleties and complexities of psychiatric entities; it also fails to consider overlapping similarities, as well as underlying mechanisms and aetiology. ${ }^{2}$

The difficulty with categorisation in psychiatry is trying to draw clear boundaries between disorders with significant heterogeneity (symptom profiles can differ significantly among patients diagnosed with the same psychiatric disorder). ${ }^{2}$ There may well also be transdiagnostic experiences that cut across diagnoses, or psychopathology that falls between existing categories. Adopting a longitudinal clinical approach allows us to observe unfolding factors associated with many conditions and the interplay between these. The phenomenological method can also be used to clarify autobiographical accounts and generate hypotheses with a focus on formulation. ${ }^{5,6}$

We have to accept that psychiatric nosology does not always map onto people all that neatly. In fact, it has been shown that important features of commonly diagnosed psychiatric disorders, such as major depression, are not always captured by DSM criteria. ${ }^{7}$ This is understandable given the complex nature of psychiatry and medicine on the whole, and a more realistic expectation of the role of categorisation may be needed. ${ }^{3}$ A complementary approach, with an understanding of the usefulness of diagnostic categories, as well as a longitudinal approach and phenomenology as clinical tools, is necessary.

\section{The literature on the shared features and comorbidity of BPAD, EUPD and ADHD}

Research points to significant overlap between personality disorders, mood disorders, and developmental disorders such as ADHD. ${ }^{8}$ EUPD, BPAD and ADHD share features of impulsivity, mood lability and relationship problems. Clinicians may be reluctant to diagnose EUPD because it is perceived as 'untreatable'.9 Longitudinal studies have found that EUPD diagnoses (despite being pervasive by definition) are not necessarily stable, and evidence-based treatment is available. ${ }^{10}$ The boundary between bipolar, particularly type 2, and EUPD is particularly porous: EUPD is often diagnosed alongside bipolar type 2. Zimmerman and Morgan ${ }^{11}$ argue that overdiagnosis of bipolar is often due to misrecognition of EUPD symptoms. Regarding the comorbidity of ADHD and $\mathrm{BPAD}$, Youngstrom and others ${ }^{12}$ demonstrate that distinguishing between 'true' and 'false' comorbidity is a complex and shifting process. EUPD symptoms can thus be conceptualised as a phenotypic variant of BPAD, related to developmental factors. ${ }^{13}$

Recent research indicates an association with similar biological mechanisms across these disorders. For example, there is evidence of hypothalamic pituitary axis dysfunction and childhood psychosocial adversity in ADHD; the same risk factors are also associated with BPAD and EUPD. ${ }^{14,15}$ There is evidence of genetic vulnerabilities to a range of mental and developmental disorders, including ADHD and BPAD. ${ }^{16-18}$ Childhood difficulties and early traumatic life experiences have also been described in a number of psychiatric conditions including EUPD, BPAD, major unipolar depression and ADHD, suggesting common underlying aetiological processes. ${ }^{19}$

The current research on these psychiatric conditions draws attention to shared aetiological pathways and suggests validated risk factors, some of which are potentially modifiable through available or novel treatments. The gap in the literature is the lack of systematic efforts to link specific risk and causal factors with the phenomenological level. This would help to further discriminate between these entities and hopefully enable the design of clinically meaningful and useful interventions. 


\section{Assessment of mental symptoms in psychiatry}

The phenomenological approach is an indispensable clinical tool that can help with assessment and interpretation of mental symptoms. It allows a detailed enquiry into their patterns of emergence, their unfolding over time and their relationship with aspects of personality. In contrast with a symptom checklist approach, phenomenology favours a dialectical approach which is semi-structured and supports the patient's narration of their personal experience. The aim is not a merely descriptive account of the patient's experiences (descriptive psychopathology); it can also offer an explanation through seeking to reveal two things: (a) the complementarity of different aspects of the patient's experiences and expressions; and (b) the temporal interdependence of distinct phases in the development of the patient's lived world. ${ }^{20}$

Take the example of affective lability or mood instability. This can take various descriptive forms, as the surrounding affect or mood can include valence, intensity, frequency of shift, rapidity of rise-times and return to baseline, reactivity to psychosocial cues and the extent to which there is overdramatic expression. ${ }^{21}$ Following a phenomenological approach (complementarity and development over time), it can also be linked with various other types of experiences, such as perception, bodily feelings, self-awareness, motivation and/or those brought about by social-cognitive processes. In EUPD, symptoms such as mood instability tend to present as temporary occurrences contingent upon situational stressors, e.g. perceived rejection and abandonment $^{22}$ and negative affect. ${ }^{23}$ Whereas there is overlap with $\mathrm{BPAD}$ in the emotion sector, experiences and expressions in EUPD also contain failures in self-appraisal, introspection and empathy (hypermentalisation). ${ }^{24}$ Regarding depression, emerging studies suggest that there are similar self and social cognition features, but they rather take the form of hypomentalisation. ${ }^{25}$ It can also be argued that in EUPD these are rather enduring trait states and probably more pervasive than in other disorders. People with depression can either have a stable low mood or also exhibit mood instability or irritability. ${ }^{26}$ The subjective experience of emotions in EUPD can have characteristics such as fusion of emotions and self and/or undifferentiated emotional experience, regularly taking the form of dissociation. It is by paying further attention to the unfolding of personal experiences that we can distinguish between depression, especially mixed affective states, and/or EUPD. In depression and mania, mood states are closely associated with cognitive states: a self-denigrated assessment that ' $\mathrm{I}$ am worthless' in depression, and an inflated sense that 'I am exceptional' in mania. ${ }^{27}$ There has not been much work on phenomenology of mood instability in ADHD and generally not much theorising about mood in clinical psychiatry.

\section{Psychiatry and complexity}

The phenomenological method is compatible with neuroscience (see work on aberrant salience and formation of delusions ${ }^{28}$ ) and can enrich both clinical practice and empirical enquiry. However, one of its main limitations is that it is a laborious, indirect and fallible inferential process that cannot give a conclusive account of psychopathological kinds unless it is combined with other levels of interpretation (risk factors, longitudinal observation, diagnosis, response to treatment, etc.). ${ }^{29}$ Psychiatry is generally characterised by complexity. A framework for understanding complex psychopathology is necessary in order to organise clinical information, plan treatment and select interventions based on both evidence and values.

A pluralistic approach, understanding the limits of diagnosis and complementing it with longitudinal observations, and using phenomenology as a method can enrich clinical practice. On a more theoretical level, which also concerns the way research can be integrated into practical psychiatry, Kendler $^{30}$ has suggested the following framework, which comprises three main guiding principles: (a) psychiatry should aim to clarify risk factors regardless of level, including those assessed using imaginative understanding and phenomenology, with careful attention to causal inference; (b) research will require tracing causal pathways across levels downward to biological neuroscience and upward to social factors, thereby elucidating the important cross-level interactions; and (c) clinicians and researchers should aim to design ways of tracing the effects of these causal pathways back up into the mental realm, moving from the level of causal explanation to that of clinical understanding.

\section{Practical management}

The management of complex patients with multiple diagnoses and/or comorbidities normally takes place in a multidisciplinary secondary care setting. Assessment should be patient centred, focusing on the nature of the presentation, the patient's values and any immediate risks, what help is being sought and whether this is available. This will inform diagnostic formulation, hypotheses and clinical investigations, and an agreed treatment plan.

Repetitive information gathering has many advantages in observing symptoms and experience over time, which is useful in conditions such as BPAD and EUPD. We do, however, need to be mindful that some patients may question the purpose of this. A clear explanation of the process of the longitudinal, in-depth assessment can help build alliance and thus improve engagement. The clinicians involved are also supported to maintain consistency (number, frequency and type of follow-ups are discussed in multidisciplinary team meetings) but also to be flexible and able to adjust to the patient's needs. This type of input is provided by the short-term community mental health team (provision of care for a maximum of 3 months), which specialises in lengthy assessments, formulations and brief interventions. The short-term team has interface links with various other teams (home treatment team, substance misuse team, long-term teams and a range of specialist services).

One of the main diagnostic/assessment tasks is to find ways to test the primacy and/or severity of a cluster of BPAD, EUPD or ADHD symptoms. For a trainee, it can be difficult to be confident in making correct diagnoses in complex cases. It is therefore important to seek help and advice where necessary, which can be through informal discussions with colleagues and multidisciplinary meetings. This provides alternative perspectives on a case as well as varied 
professional expertise. If direct contact with a patient is needed, clinical supervisors can offer support with this.

Tools that can help test diagnostic hypotheses in relation to EUPD and BPAD include the Structured Clinical Interview for DSM-5, a semi-structured interview guide that can be used to screen for personality disorders, and the Mood Disorder Questionnaire for BPAD. Questionnaires to assess severity of either EUPD or ADHD can also be useful. The conceptualisation of personality disorders is particularly important for community mental health teams. The model that we use is the integrative framework proposed by John Livesley, ${ }^{31}$ which differentiates between clinical assessment of traits and domains of psychopathology such as core self and interpersonal problems, as well as specific symptoms and failures of the regulation/modulation systems. A mood diary is a practical, simple tool that can be used to help with tracking changes in mood but also engages patients and involves them in their care. It also encourages their self-assessment and introspection, which can be further probed by semi-structured clinical interviews. Other ways of testing clinical hypotheses include longitudinal observation, although this is not always practical owing to limitations such as large clinical caseloads and pressures to reduce these. Trainees may find it indispensable to explore techniques for eliciting information in interviews, discuss relevant research findings and address risk factors in supervision and within the team to enrich exploration of relevant information that can contribute to formulation.

In this case, the patient had established diagnoses of ADHD, EUPD and BPAD. As we improved our knowledge of the patient and begin to develop our case formulation based on the methods explained above, the diagnosis of EUPD was questioned and later discarded, which highlights some of the issues discussed in this paper. Defining personality disorder can be a daunting task. In community psychiatry, we tend to distinguish personality disorder from the far more common personality dysfunction. This is based on an assessment of severity, as well as exploration of markers that can be used to differentiate an organised from a disorganised personality system (unstable sense of self, chronic interpersonal dysfunction). ADHD and BPAD can also affect relationships with the self and others. In these cases, there can be benefit from psychotherapy, which might help the person to understand their illness and relationships, although the exact type of psychotherapy offered may be influenced by whether they have been formally diagnosed with a disorder or dysfunction.

It was felt that we needed to identify a helpful mode of pharmacological therapy that would tackle the patient's BPAD symptoms in order to provide some stability and allow exploration and addressing of other factors (cognitive symptoms, self-efficacy, relationships, etc.). This would also help to test the hypothesis relevant to comorbid, untreated ADHD: that cognitive acceleration reduces if the mood is stabilised. In this case, the patient had already been trialled on mood stabilisers, but these were discontinued owing to side-effects. For a trainee, this can be a difficult situation to be faced with when first- and second-line treatment options have already been ruled out. It is always worth keeping an open mind when exploring past pharmacological intervention as to why treatments didn't work. This may allow re-visitation of failed treatments in a clinical context, e.g. if mania had been triggered by certain antidepressants, supporting a diagnosis of BPAD.

Finally, a long-term view is necessary to capture the evolving nature of $\mathrm{BPAD}$, to help disentangle and address comorbidity (psychiatric and physical) and to improve outcomes. Furthermore, a longitudinal approach is essential to elucidate the underlying biology of BPAD from high-risk individuals and prodromal presentations to established cases. ${ }^{32}$ In this case, the short-term team provided eight sessions, which helped to provide a diagnostic formulation and treat acute mixed affective symptoms. We then decided to refer to a longterm team, as we thought a longitudinal approach would be necessary owing to the complexity and the chronicity of some of the issues discussed here.

The patient herself reflects thus on the experience:

My treatment at South London and Maudsley NHS Foundation Trust was a positive experience overall, since in the past during treatment with previous clinicians I had felt dismissed as an example of 'untreatable' EUPD. I felt that my complex presentation was dealt with in combination rather than as a stand-alone personality disorder. The less helpful aspect of treatment was something I find common to psychiatric treatment, such as a focus on medication alone rather than as a course of more 'holistic' treatment including, for example, therapy suggesting coping strategies (but austerity-related cuts leading to long waiting lists for therapy contribute to this issue).

\section{Conclusions}

In this case report, we address the problem of comorbidity and complexity in psychiatry. We synthesise the perspectives and views of a general practice trainee, two consultant psychiatrists and a person with lived experience. We acknowledge the significant overlap of personality disorders, mood disorders, and developmental disorders such as ADHD. We then discuss the pros and cons of diagnostic categorisation and suggest that diagnostic formulation can be combined with longitudinal observation and phenomenology. We also consider the importance of informing clinical work and therapeutic relationships with discussions about complexity, shared aetiological pathways and awareness of risk factors. We suggest a person-centred, longitudinal approach that endorses pluralism and is aware of its limitations.

\section{Author contributions}

J.W. and R.C. drafted the first version of the manuscript. D.A. and M.K. contributed to all the versions of the manuscript. M.K. developed the idea for the article and designed the structure. All the authors approved the final version of the manuscript.

\section{About the authors}

Jonathan Williams is a GP trainee at the Guy's and St Thomas' General Practitioner Vocational Training Scheme, London, UK. Ruth Cain is a Senior Lecturer at the School of Law, University of Kent, Eliot College Extension, University of Kent, UK. Danilo Arnone is an Associate Professor of Psychiatry and Consultant Psychiatrist at the Department of Psychiatry, College of Medicine and Health Sciences, United Arab Emirates University, United Arab Emirates and in the Department of Psychological Medicine, Institute of Psychiatry, Psychology \& Neuroscience, King's College London, UK. Michalis Kyratsous is a Consultant Psychiatrist at 
South London and Maudsley NHS Foundation Trust and an MD(Res) student at the Department of Psychological Medicine, Institute of Psychiatry, Psychology and Neuroscience, King's College London, UK.

\section{References}

1 World Health Organization. International Statistical Classification of Diseases and Related Health Problems (10th revision). WHO, 1992.

2 Moses J. Psychiatric diagnosis and the medical model: a critique of a suggested revision. PsycCRITIQUES 2017; 62(35): 3.

3 Nesse R, Stein D. Towards a genuinely medical model for psychiatric nosology. BMC Med 2012; 10. Available from: https://doi.org/10.1186/ 1741-7015-10-5.

4 Sedler M. Medicalization in psychiatry: the medical model, descriptive diagnosis, and lost knowledge. Med Health Care Philos 2015; 19(2): 247-52

5 Ratcliffe M. Understanding existential changes in psychiatric illness: the indispensability of phenomenology. Psychiatry Cogn Neurosci 2009: 221-44.

6 Seising R. From vagueness in medical thought to the foundations of fuzzy reasoning in medical diagnosis. Artif Intell Med 2006; 38(3): 237-56.

7 Kendler K. The phenomenology of major depression and the representativeness and nature of DSM criteria. Am J Psychiatry 2016; 173(8) 771-80.

8 Magill C. The boundary between borderline personality disorder and bipolar disorder: current concepts and challenges. Can J Psychiatry 2004: 49(8): 551-6.

9 Paris J. Why psychiatrists are reluctant to diagnose: borderline personality disorder. Psychiatry 2007; 4(1): 35-9.

10 Gunderson J. Ten-year course of borderline personality disorder. Arch Gen Psychiatry 2011; 68(8): 827-37.

11 Zimmerman M, Morgan T. Problematic boundaries in the diagnosis of bipolar disorder: the interface with borderline personality disorder. Curr Psychiatry Rep 2013; 15(12): 422.

12 Youngstrom E, Arnold L, Frazier T. Bipolar and ADHD comorbidity: both artifact and outgrowth of shared mechanisms. Clin Psychol Sci Pract 2010; 17(4): 350-9.

13 Perugi G, Angst J, Azorin J, Bowden C, Vieta E, Young A. Is comorbid borderline personality disorder in patients with major depressive episode and bipolarity a developmental subtype? Findings from the international BRIDGE study. J Affect Disord 2013; 144(1-2): 72-8.

14 Kulacaoglu F, Solmaz M, Ardic F, Akin E, Kose S. The relationship between childhood traumas, dissociation, and impulsivity in patients with borderline personality disorder comorbid with ADHD. Psychiatry Clin Psychopharmacol 2017; 27(4): 393-402.

15 Richard-Lepouriel H, Kung A, Hasler R, Bellivier F, Prada P, Gard S, et al. Impulsivity and its association with childhood trauma experiences across bipolar disorder, attention deficit hyperactivity disorder and borderline personality disorder. J Affect Disord 2019; 244: 33-41.
16 Identification of risk loci with shared effects on five major psychiatric disorders: a genome-wide analysis. Lancet 2013; 381(9875): 1371-9.

17 Faraone S, Glatt S, Tsuang M. The genetics of pediatric-onset bipolar disorder. Biol Psychiatry 2003; 53(11): 970-7.

18 Kolla N, Meyer J, Sanches M, Charbonneau J. Monoamine oxidase-a genetic variants and childhood abuse predict impulsiveness in borderline personality disorder. Clin Psychopharmacol Neurosci 2017; 15(4): 343-51.

19 Carr C, Martins C, Stingel A, Lemgruber V, Juruena M. The role of early life stress in adult psychiatric disorders. J Nerv Ment Dis 2013; 201(12): 1007-20.

20 Gallagher S, Schmicking D. Handbook of Phenomenology and Cognitive Science: 635-54. Springer, 2010.

21 Broome M, Saunders K, Harrison P, Marwaha S. Mood instability: significance, definition and measurement. Br J Psychiatry 2015; 207(4): 283-5.

22 Miskewicz $K$, Fleeson $W$, Arnold $E$, Law $M$, Mneimne $M$, Furr $R$. A contingency-oriented approach to understanding borderline personality disorder: situational triggers and symptoms. J Pers Disord 2015; 29(4): 486-502.

23 Law M, Fleeson W, Arnold E, Furr R. Using negative emotions to trace the experience of borderline personality pathology: interconnected relationships revealed in an experience sampling study. J Pers Disord 2016; 30(1): 52-70.

24 Gunderson J, Fruzzetti A, Unruh B, Choi-Kain L. Competing theories of borderline personality disorder. J Pers Disord 2018; 32(2): 148-67.

25 Bateman A, Fonagy P. Borderline personality disorder and mood disorders: mentalizing as a framework for integrated treatment. J Clin Psychol 2015; 71: 792-804.

26 Bowen R, Peters E, Marwaha S, Baetz M, Balbuena L. Moods in clinical depression are more unstable than severe normal sadness. Front Psychiatry 2017; 8: 56

27 Radden J. The self and its moods in depression and mania. J Conscious Stud 2013; 20(7-8): 7-8.

28 Kapur S. Psychosis as a state of aberrant salience: a framework linking biology, phenomenology, and pharmacology in schizophrenia. Am Psychiatry 2003; 160(1): 13-23.

29 Oulis P. The epistemological role of empathy in psychopathological diagnosis: a contemporary reassessment of Karl Jaspers' account. Philos Ethics Humanit Med 2014; 9(1): 6.

30 Kendler K. The structure of psychiatric science. Am J Psychiatry 2014; 171(9): 931-8.

31 Livesley W. Integrated Modular Treatment for Borderline Personality Disorder. Cambridge University Press, 2017.

32 Cross SP, Hermens DF, Scott EM, Ottavio A, McGorry PD, Hickie IB. A clinical staging model for early intervention youth mental health services. Psychiatr Serv 2014; 65(7): 939-43.

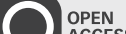

